\title{
The Araucaria Project: the Baade-Wesselink projection factor of pulsating stars and interferometric contribution to the eclipsing binaries technique of distance determination
}

\author{
Nicolas Nardetto, ${ }^{1}$ Mounir Challouf, ${ }^{1,2}$ Guillaume Guiglion, ${ }^{1}$ \\ Denis Mourard, ${ }^{1}$ Wolfgang Gieren, ${ }^{3}$ Grzegorz Pietrzyński, ${ }^{3,4}$ and \\ Dariusz Graczyk ${ }^{3}$ \\ ${ }^{1}$ Laboratoire Lagrange, UMR7293, UNS/CNRS/OCA, 06300 Nice, France \\ email: Nicolas. Nardetto@oca.eu \\ ${ }^{2}$ Lab. Dyn. Moléculaire et Matériaux Photoniques, UR11ES03, UT2/ESSTT, Tunis, Tunisia \\ ${ }^{3}$ Universidad de Concepción, Departamento de Astronomía, Casilla 160-C, Concepción, Chile \\ ${ }^{4}$ Warsaw University Observatory, Al. Ujazdowskie 4, 00-478, Warsaw, Poland
}

\begin{abstract}
The international Araucaria Project aims to provide an improved local calibration of the extragalactic distance scale out to distances of a few Megaparsecs. We present a brief, up-to-date review of different results and ongoing projects to (i) better understand the BaadeWesselink projection factor used in distance determinations based on Cepheids, and (ii) improve the eclipsing-binary technique of distance determination using CHARA/VEGA interferometric observations.
\end{abstract}

Keywords. stars: oscillations, stars: distances, binaries: eclipsing

\section{Introduction}

In setting up the extragalactic distance scale, the greatest difficulty leading to the currently largest contribution to the systematic uncertainty in the Hubble constant resides in the determination of accurate absolute distances to nearby galaxies. The Araucaria Project† (Gieren et al. 2005) is an effort to remedy this situation for several of the most important stellar candles, including Cepheid variables, RR Lyrae stars, red clump giants, blue supergiants, and eclipsing binaries, which all have the potential to provide accurate distance determinations to nearby galaxies. In this paper, we focus on recent results and ongoing projects regarding the Baade-Wesselink projection factor for Cepheids (and other types of pulsating stars) and on the eclipsing-binary technique.

\section{The Baade-Wesselink projection factor}

The projection factor, $p$, used in Baade-Wesselink-type methods of Cepheid distance determinations links stellar physics with the cosmological distance scale. A coherent picture of this physical quantity is now provided based on high-resolution spectroscopy and hydrodynamical modelling (Nardetto et al. 2004, 2007, 2009). However, recent observations of 36 Cepheids in the Large Magellanic Cloud (LMC) provide new constrains to the projection factor, and a discrepancy is found for short-period Cepheids (Storm et al. 2011a,b). For instance, for $\delta$ Cep, Storm et al. find $p=1.41 \pm 0.05$, while the theoretical 
value is $p=1.25 \pm 0.05$ (Nardetto et al. 2009). An effect of the physical nature of LMC Cepheids on the slope of the period-projection-factor $(P p)$ relation cannot be excluded. Nevertheless, it has been shown theoretically that metallicity does not affect the value of the projection factor (Nardetto et al. 2011). Similarly, the weak non-linearity found in the $P p$ relation (Neilson et al. 2012; Ngeow et al. 2012) cannot explain such a $(3 \sigma)$ discrepancy.

We try to resolve this discrepancy through a global approach, by exploring the projection factor for different kinds of pulsating stars in the Hertzsprung-Russell diagram. We focus first on $\delta$ Scuti and $\beta$ Cephei stars. Such pulsating stars have shorter periods than Cepheids (typically a few hours compared to a few days). We adopt the same strategy as for Cepheids using hydrodynamical models (Fokin et al. 2004) and high spectralresolution observations. Here we emphasize two main results:

- In Guiglion et al. (2012) we studied mainly two $\delta$ Scuti stars, i.e., the high-amplitude pulsator AI Vel and the fast rotator $\beta$ Cas, using ESO/HARPS and OHP/SOPHIE spectroscopic data, respectively. We compared the radial velocity curves of several spectral lines that form at different levels in the atmosphere to determine whether the atmospheric velocity gradient could affect the projection factor. We found that the atmospheric velocity gradient (where the spectral lines are formed) is essentially zero and that the $P p$ relation derived for Cepheids by Nardetto et al. (2007) is also applicable to $\delta$ Scuti stars. Nevertheless, we also found that for relatively fast rotating stars like $\beta$ Cas $(\simeq 70 \mathrm{~km}$ $\left.\mathrm{s}^{-1}\right)$, the projection factor depends significantly on the inclination of the rotation axis $(i)$ because of the Van Zeipel gravity darkening effect of the star's intensity distribution (see Fig. 1). Such an effect (owing to the random orientation of the rotation axis of $\delta$ Scuti stars) migh explain the relative dispersion in the $P p$ relation of $\delta$ Scuti stars found by Laney \& Joner (2009). In this study we also provide a brief discussion of the impact of non-radial modes on the projection factor.

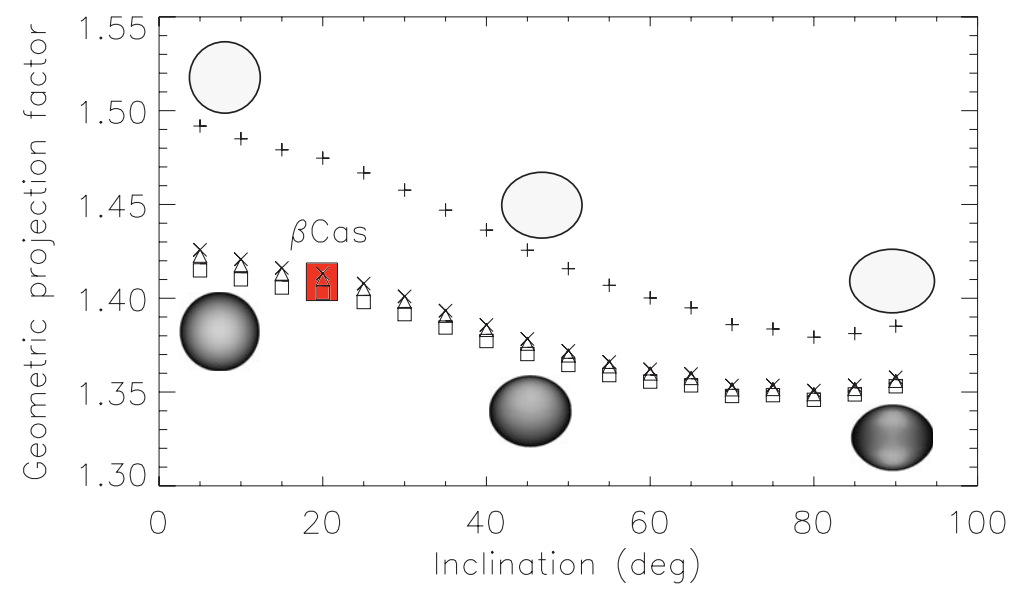

Figure 1. Geometric projection factor, $p_{0}$ (i.e., not including the dynamical structure of the atmosphere), as a function of inclination for the rapidly rotating star $\beta$ Cas at three different wavelengths: $\lambda=6000 \AA(\square), \lambda=6500 \AA(\triangle)$, and $\lambda=7000 \AA(\times)$. The red box indicates the uncertainty in $p_{0}$. The case of a uniform, elongated disk is overplotted $(+)$ and we find that $p_{0}=1.5$ for $i=0^{\circ}$, as expected for a circular, uniform disk. These intensity maps are based on the code by Domiciano de Souza et al. (2002).

- In Nardetto et al. (2012) we focus on the $\beta$ Cephei star $\alpha$ Lup and, again using HARPS spectroscopic data, we find - similarly to $\delta$ Scuti stars - no atmospheric velocity gradient. Despite the absence of an atmospheric velocity gradient, and because of subtle 
physical effects (probably related to radiative-transfer effects), the final theoretical value of the projection factor is much lower than expected from Nardetto et al. (2007), by approximately $10 \%$. Further investigation will be necessary to fully understand this result. Nevertheless, we clearly found (directly from observations) a very interesting relation between the amplitude of the radial velocity curve and the atmospheric velocity gradient.

By comparing Cepheids, $\delta$ Scuti, and $\beta$ Cephei stars, these results offer valuable insights into the dynamical structure of pulsating stellar atmospheres, which help to understand the $k$-term problem (Nardetto et al. 2008) and the projection factor for Cepheids.

\section{Interferometric contribution to the eclipsing-binary technique of distance determination}

Eclipsing binaries are currently used to derived the distance to the LMC with an unprecedented accuracy of $2 \%$ (Graczyk et al. 2012; Pietrzyński et al. 2012b). They are also extremely interesting for the derivation of the component masses (Pietrzyński et al. 2010, 2011, 2012).

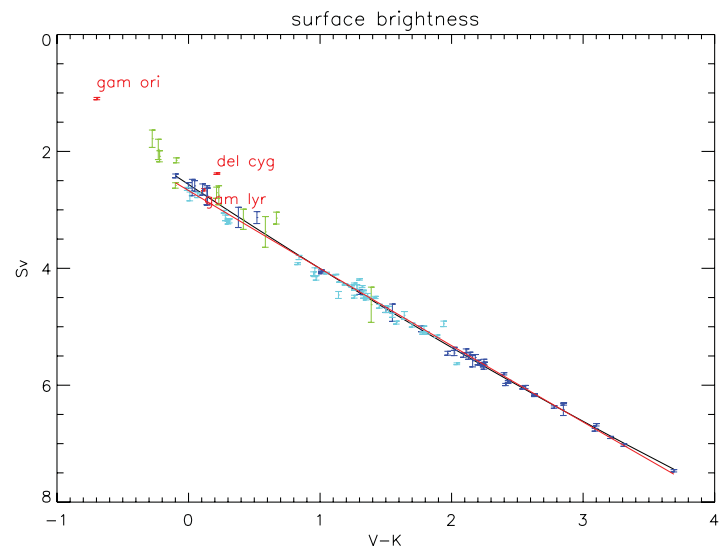

Figure 2. Visual surface-brightness parameter $S_{\mathrm{v}}$ as a function of $(V-K)$. The dark blue measurements represent the angular diameters of the 44 stars of Di Benedetto (2005). The light blue points indicate the angular diameters of the 44 stars determined by Boyajian et al. (2012). The black line is the fit by Di Benedetto and the red line is the fit using the measurements of Di Benedetto and Boyajian et al. The red points are the recent CHARA/VEGA interferometric measurements of $\gamma$ Ori, $\gamma$ Lyr, and $\delta$ Cyg.

This method of distance determination consists of combining the radii of the stars determined from spectrophotometric observations with their angular diameters derived from the surface brightness (SB) relation. Currently, the largest limitation to the method's applicability is the uncertainty in the SB relationship. Increasing the accuracy of this relation for FGK stars (by a factor of 2 ) would allow reaching a $1 \%$ precision in the distance to the LMC. In addition, constraining the SB relation for B stars would allow the use of brighter eclipsing-binary systems, and thus help to increase the number of suitable, distant objects. The aim of our ongoing work is to improve our understanding of the SB relationship (especially for B stars) using interferometry (Challouf et al. 2012).

The VEGA recombiner operates in the optical (Mourard et al. 2009) and benefits from the CHARA baselines (up to $300 \mathrm{~m}$ ). With an angular resolution of 0.3 mas, it allows precise determination of angular diameters. Fig. 2 presents preliminary results regarding the calibration of the SB relation. The capabilities of VEGA are shown in the 
context of the relative precision in the angular diameter of $1 \%$ for the three stars already observed. Including recent results by Boyajian et al. (2012), we find that the relation by Di Benedetto (2005) is slightly modified and the precision improved. However, more work is needed to select a sample of stars carefully, depending on their class and fundamental characteristics, to avoid any biases. In particular for B stars, the impact of a stellar wind cannot be excluded and will be studied. Such a study will be possible only with a larger sample of B stars. In total, we expect to observe 10 more stars with the CHARA/VEGA interferometer. Some observations are already ongoing. (This work is part of the Ph.D. thesis research of M. Challouf.)

Although they relate to very different astrophysical objects such as Cepheids, $\delta$ Scuti, $\beta$ Cephei stars, and eclipsing binaries, the studies presented in this brief contribution, aim to improve the local calibration of the extragalactic distance scale, and in particular the distance to the LMC. Our goal is to achieve $1 \%$ precision to the LMC based on these independent methods.

\section{Acknowledgements}

WG and GP gratefully acknowledge financial support for this work from the Chilean Center for Astrophysics FONDAP 15010003, and from the BASAL Centro de Astrofísica y Tecnologías Afines (CATA) PFB-06/2007. Support from Polish grant N203 387337 and a TEAM subsidy of the Fundation for Polish Science (FNP) are also acknowledged. This research received support from PHASE, the high angular resolution partnership between ONERA, Observatoire de Paris, CNRS, and the University Denis Diderot Paris 7. The authors thanks H. Aroui, E. Chapellier, O. Chesneau, A. Domiciano De Souza, A. Fokin, P. Mathias, and E. Poretti who also contributed to these results.

\section{References}

Di Benedetto, G. P. 2005, MNRAS, 357, 174

Boyajian, S., McAlister, A., van Belle, G., et al. 2012, ApJ, 746, 101

Challouf, M., Nardetto, N., Mourard, D., et al. 2012, Proc. SF2A Conf., submitted

Domiciano de Souza, A., Vakili, F., Jankov, S., et al. 2002, A\&A, 393, 345

Gieren, W., Pietrzyński, G., Bresolin, F., et al. 2005, ESO Messenger, 121, 23

Graczyk, D., Pietrzyński, G., Thompson, I. B., et al. 2012, ApJ, 750, 144

Fokin, A., Mathias, P., Chapellier, E., et al. 2004, A\&\&A, 426, 687

Guiglion, G., Nardetto, N., et al. 2012, A\&BA, submitted

Laney, C. D. \& Joner, M. D., 2009, in: AIP Conf. Ser. (Guzik, J.A., \& Bradley, P.A., eds), 1170, p. 93

Mourard, D., Clausse, J. M., Marcotto, A., et al. 2009, A\& $A$, 508, 1073

Nardetto, N., Fokin, A., Mourard, D., et al. 2004, A\&A, 428, 131

Nardetto, N., Mourard, D., Mathias, P., et al. 2007, A\&A, 471, 661

Nardetto, N., Stoekl, A., Bersier, D., et al. 2008, A\&A, 489, 1255

Nardetto, N., Gieren, W., Kervella P., et al. 2009, A\&A, 502, 951

Nardetto, N., Fokin., A., Fouqué, P., et al. 2011, A\&A, 534, 16

Nardetto, N., Mathias, P., Fokin, A., et al. 2012, A\&A, submitted

Ngeow, C.-C., Neilson, H. R., Nardetto, N., et al. 2012, A\&BA, 543, 55

Neilson, H. R., Nardetto, N., Ngeow, C.-C., et al. 2012, A\&A A, 541, 134

Pietrzyński, G., Thompson, I. B., Gieren, W., et al. 2010, Nature, 468, 542

Pietrzyński, G., Thompson, I. B., Graczyk, D., et al. 2011, ApJ, 742, 20

Pietrzyński, G., Thompson, I. B., Gieren, W., et al. 2012a, Nature, 484, 75

Pietrzyński, G., Gieren W., Thompson, I. B., et al. 2012b, Nature, submitted

Storm, J., Gieren, W., Fouqué, P., et al. 2011a, A\& A, 534, 94

Storm, J., Gieren, W., Fouqué, P., et al. 2011b, A\& $A$, 534, 95 